\title{
Point source contamination in CMB non-Gaussianity analyses
}

\author{
Daniel Babich* \\ Theoretical Astrophysics, California Institute of Technology, Pasadena, California 91125 USA \\ Elena Pierpaoli ${ }^{+}$ \\ University of Southern California, Los Angeles, California 90089 USA \\ (Received 27 March 2008; published 23 June 2008)
}

\begin{abstract}
In this paper we analyze the biasing effect of point sources, either thermal Sunyaev-Zeldovich clusters or standard radio sources, on the estimated strength of the non-Gaussianity in the cosmic microwave background (CMB). We show that the biggest contribution comes from the cross correlation of the CMB with the matter density rather than from the Poisson term which is conventionally assumed in these calculations. For the three year WMAP data, we estimate that point sources could produce a non-Gaussian signature equivalent to a bias in $f_{N L}$ of $0.35,0.24,-0.097,-0.13$ in the $\mathrm{Ka}, \mathrm{Q}, \mathrm{V}$, and $\mathrm{W}$ bands, respectively. The level of bias we find is largely insufficient to explain the very high $f_{N L}$ values recently detected by Yadav and Wandelt. For Planck, we estimate the point source bispectra to contaminate the $f_{N L}$ estimator with a bias of $1.3,0.34,-0.25,-0.48$ at $30,44,70,100 \mathrm{GHz}$, respectively. These results depend on the assumed redshift distribution of the point sources. However, given the projected Planck sensitivity of $\Delta f_{N L} \simeq 5$ (95\% C.L.), a good estimate of point sources' properties including their number density and redshift distribution is essential before deriving strong conclusions on primordial non-Gaussianity.
\end{abstract}

DOI: 10.1103/PhysRevD.77.123011

PACS numbers: 98.70.Dk, 98.80.-k

\section{INTRODUCTION}

Recent claims by Yadav and Wandelt [1] of the detection of strong primordial non-Gaussianity in the three year Wilkinson Microwave Anisotropy Probe (WMAP) data [2] have the potential to revolutionize our understanding of the early universe. These results were also found in the WMAP five year analysis, although with less statistical significance [3]. The strength of the non-Gaussianity detected in their analysis is more than 2 orders of magnitude larger than the non-Gaussianity expected in the simplest model of the single field, slow roll model of inflation [4]. This detection, if it stands up to scrutiny, will be the first definitive indication that the simplest model of inflation cannot adequately explain all of the current cosmological observations and must be modified in some way.

In Yadav and Wandelt's analysis [1], the detection of the non-Gaussian signal is due to the simultaneous reduction in the estimator's error bars and the shift in the central value as smaller angular scale information was included in the analysis. One obvious concern is that the estimator is contaminated by foreground emission, in particular, radio points sources and the thermal Sunyaev-Zeldovich (SZ) effect which become increasingly more important on small angular scales.

In this paper we will analyze the influence of point sources, for the experiment resolution of WMAP both the radio sources and SZ clusters effectively act as point sources, on the standard non-Gaussianity estimator.

\footnotetext{
*babich@tapir.caltech.edu

${ }^{+}$pierpaol@usc.edu
}

While it has been claimed that the non-Gaussianity caused by Poisson fluctuations in the number density of radio point sources can be safely separated from the primordial non-Gaussian signal [5], we will demonstrate the other forms of point source non-Gaussianity cannot be safely ignored. In addition to the standard forms of nonGaussianity produced by point sources, we will show that cross correlation between the point source power spectrum and the cosmic microwave background (CMB) temperature anisotropies or instrument noise will produce nonGaussianity of a very similar form as the local model. The local model is the form typically assumed in analyses of primordial non-Gaussianity, so these new non-Gaussian contributions will bias the estimator in a fashion that cannot easily be corrected.

This paper is organized as follows. In Sec. III we demonstrate that point sources can produce a bispectrum that has the same form as the local model. In Sec. II we derive the bias induced by the various point source bispectra. In Sec. VI we conclude. We use the WMAP three year cosmological model [2] for numerical calculations.

\section{ESTIMATOR BIAS}

While non-Gaussianity generically implies that any higher-order connected correlation function is nonzero, it is typical to focus on the three-point correlation function, or equivalently the bispectrum, because it has the simplest form of all non-Gaussian correlation functions and for weak non-Gaussianity it contains nearly all of the information [6]. The three-point correlation function can be factored into a component fixed by rotational invariance, 
which is assumed a prioiri, and a piece determined by the underlying mechanism that produced the non-Gaussianity [5]. Rotational invariance forces the three-point correlation function to be proportional to the Gaunt integral,

$$
\begin{aligned}
\mathcal{G}_{m_{1} m_{2} m_{3}}^{\ell_{1} \ell_{2} \ell_{3}}= & \sqrt{\frac{\left(2 \ell_{1}+1\right)\left(2 \ell_{2}+1\right)\left(2 \ell_{3}+1\right)}{4 \pi}} \\
& \times\left(\begin{array}{ccc}
\ell_{1} & \ell_{2} & \ell_{3} \\
m_{1} & m_{2} & m_{3}
\end{array}\right)\left(\begin{array}{ccc}
\ell_{1} & \ell_{2} & \ell_{3} \\
0 & 0 & 0
\end{array}\right),
\end{aligned}
$$

and the CMB three-point correlation function can be written as

$$
\left\langle a_{\ell_{1} m_{1}} a_{\ell_{2} m_{2}} a_{\ell_{3} m_{3}}\right\rangle=G_{m_{1} m_{2} m_{3}}^{\ell_{1} \ell_{2} \ell_{3}} b_{\ell_{1}, \ell_{2}, \ell_{3}},
$$

where the reduced bispectrum, $b_{\ell_{1}, \ell_{2}, \ell_{3}}$, contains information about the form of non-Gaussianity.

The estimators used in CMB non-Gaussianity analyses are optimized for the detection of a signal with a very particular form, namely, the local model [6]. The local model assumes that the initial curvature perturbations can be written as

$$
\Phi(\boldsymbol{x})=\phi_{g}(\boldsymbol{x})+f_{N L}\left[\phi_{g}^{2}(\boldsymbol{x})-\left\langle\boldsymbol{\phi}_{g}^{2}(\boldsymbol{x})\right\rangle\right],
$$

where $\phi_{g}$ is Gaussian. The nonlinear terms in this model lead to the following bispectrum for the initial curvature perturbations

$$
B\left(k_{1}, k_{2}, k_{3}\right)=2 f_{N L}\left[P\left(k_{1}\right) P\left(k_{2}\right)+\mathrm{cyc}\right],
$$

where $P(k)$ is the power spectrum. The ordinary linear radiative transfer functions are subsequently used to calculate the CMB temperature anisotropies from these initial curvature perturbations. The statistical properties of the CMB temperature anisotropies will mirror the statistical properties of underlying curvature perturbations since we consider linear radiative transfer. The levels of nonGaussianity claimed by Yadav and Wandelt are significantly larger than the expected non-Gaussianity produced by nonlinear radiative transfer.

However the estimators are sensitive to any bispectrum form that might be present in the data, regardless of its origin. In this section we will determine the induced bias produced by the various cross correlation bispectra described in the next section.

The non-Gaussianity estimator can be expressed as [7]

$$
\hat{f}_{N L}=\frac{1}{A}\left[\sum G_{m_{1} m_{2} m_{3}}^{\ell_{1} \ell_{2} \ell_{3}} \frac{b_{\ell_{1}, \ell_{2}, \ell_{3}}}{C_{\ell_{1}}^{T} C_{\ell_{2}}^{T} C_{\ell_{3}}^{T}} a_{\ell_{1} m_{1}} a_{\ell_{2} m_{2}} a_{\ell_{3} m_{3}}\right] \text {, }
$$

where the normalization is

$$
\begin{aligned}
A= & \sum \frac{\left(2 \ell_{1}+1\right)\left(2 \ell_{2}+1\right)\left(2 \ell_{3}+1\right)}{4 \pi}\left(\begin{array}{ccc}
\ell_{1} & \ell_{2} & \ell_{3} \\
0 & 0 & 0
\end{array}\right)^{2} \\
& \times \frac{b_{\ell_{1}, \ell_{2}, \ell_{3}}^{2}}{C_{\ell_{1}}^{T} C_{\ell_{2}}^{T} C_{\ell_{3}}^{T}},
\end{aligned}
$$

TABLE I. Radio point source flux threshold, FWHM, and instrument pixel noise (in $10^{-6}$ ) for the relevant WMAP and Planck frequency bands. For WMAP the various band names are also listed.

\begin{tabular}{llcr}
\hline \hline Frequency $(\mathrm{GHz})$ & $\bar{S}(\mathrm{Jy})$ & FWHM (arcmin) & $\Delta T / T$ \\
\hline WMAP-33 (Ka) & 0.7 & 41 & 5.7 \\
WMAP-41 (Q) & 0.7 & 28 & 8.2 \\
WMAP-61 (V) & 0.7 & 21 & 11.0 \\
WAMP-94 (W) & 0.7 & 13 & 18.3 \\
Planck-30 & 0.33 & 33 & 1.6 \\
Planck-44 & 0.36 & 23 & 2.4 \\
Planck-70 & 0.34 & 14 & 3.6 \\
Planck-100 & 0.13 & 11 & 1.6 \\
\hline \hline
\end{tabular}

here $C_{\ell}^{T}=C_{\ell}+C_{\ell}^{N}$ is the sum of the CMB signal and noise. The CMB experimental noise parameters are described in Table I.

We are ignoring the additional linear term in the estimator because the contribution of radio point source will not bias this piece of the estimator if the signal and noise covariance matrices well represent the real data.

The weight functions used in the estimator are optimized for a bispectrum produced by the local model. The estimator bias will be determined by substituting the various forms of the point sources' bispectra into the estimator

$$
\begin{aligned}
\Delta f_{N L}^{\alpha}= & \frac{1}{A} \sum \frac{\left(2 \ell_{1}+1\right)\left(2 \ell_{2}+1\right)\left(2 \ell_{3}+1\right)}{4 \pi} \\
& \times\left(\begin{array}{ccc}
\ell_{1} & \ell_{2} & \ell_{3} \\
0 & 0 & 0
\end{array}\right)^{2} \frac{b_{\ell_{1}, \ell_{2}, \ell_{3}} b_{\ell_{1}, \ell_{2}, \ell_{3}}^{\alpha}}{C_{\ell_{1}}^{T} C_{\ell_{2}}^{T} C_{\ell_{3}}^{T}} .
\end{aligned}
$$

Here $b_{\ell_{1}, \ell_{2}, \ell_{3}}^{\alpha}$ is one of the reduced bispectrum produced by point sources. The CMB bispectrum produced by the local model is generally negative. The collapsed triangle modes, which have the highest signal-to-noise, are always negative. So a positive point source bispectrum will cause a negative estimator bias. We will now discuss the possible bispectrum terms.

\section{POINT SOURCE BISPECTRA}

The observed signal is the sum of the primordial and secondary temperature anisotropies, foreground emission, and instrument noise. While the secondary anisotropies and extra-galactic foregrounds, which maybe quite nonGaussian, are important on small angular scales, the signal on the large angular scales relevant for WMAP is dominated by primary anisotropies. Thus it is assumed that any measured non-Gaussianity by WMAP is primordial in nature. We will argue that cross correlations between some of these signals may induce bispectra on large angular scales.

Moreover these bispectra may have similar forms as the local model if the signal power spectrum becomes spatial 
inhomogeneous in a manner that then correlates with a second component in the observed signal. For example, the matter overdensity will bias the local radio point source power spectrum and it will be correlated with the CMB temperature anisotropies produced via the integrated Sachs-Wolfe (ISW) effect. This will produce a bispectrum similar in form to the local model.

This is not a coincidence as the non-Gaussianity in the local model is produced by the modulation of the small scale inflaton power spectrum by the large scale inflaton fluctuations that have already left the horizon and frozen out. The parameter $f_{N L}$ is a measure of the nonlinear coupling between these different scales. Likewise the large scale matter overdensity modulates the small scale Poisson fluctuation power spectrum by altering the local number density of radio point sources. In an analogous fashion the bias describes the coupling between the different scales. As discussed in the previous section any bispectrum present in the data can bias the estimator.

\section{ANISOTROPY MECHANISMS}

Now we will discuss the various physical effects considered in this paper-radio point source emission, the thermal Sunyaev-Zeldovich effect, and the integrated Sachs-Wolfe effect.

\section{A. Radio point sources}

The temperature anisotropy induced by unresolved radio point sources can be expressed as an integral over their flux distribution function

$$
\frac{\Delta T}{T}(\hat{\boldsymbol{n}}, \nu)=\frac{1}{c_{\nu}} \int_{0}^{\bar{S}(\hat{\boldsymbol{n}})} d S S \frac{d N}{d S}(S, \nu ; \hat{\boldsymbol{n}}) .
$$

The conversion between the temperature and intensity fluctuations is

$$
c_{\nu}=\frac{\partial B_{\nu}}{\partial \ln T}\left(T_{\mathrm{CMB}}\right),
$$

where $T_{\mathrm{CMB}}=2.728 \mathrm{~K}$ and $B_{\nu}(T)$ is a blackbody frequency distribution.

Note that we have allowed both the upper flux limit for unresolved radio point sources and their number density to be spatially inhomogeneous. As we will describe below, these spatial inhomogeneities will correlate with other signals present in the data to produce bispectra in the observed CMB data.

The actual values for the bispectra calculated in this paper depend on the radio point sources' properties (both flux and redshift distributions) and on the flux cut at a given frequency for a specific experiment. In order to evaluate the residual point source contribution to the total estimated bispectrum in the WMAP-three year's data, we must consider the technique used by WMAP to identify and substract radio point sources [8] and the point source mask applied to the data. The WMAP source selection criterion does not correspond to a single flux threshold at a given frequency, rather it requires that a candidate source should be seen with a minimal statistical significance in all channels [9]. As Yadav and Wandelt's results [1], which are the motivation for this paper, were derived using the $\mathrm{V}$ and $\mathrm{W}$ frequency bands, the radio point source populations at these frequencies is the most relevant. Unfortunately, as most radio sources are stronger at low frequencies, they tend to be detected with higher significance in the $\mathrm{K}-\mathrm{Q}$ bands than in the $\mathrm{V}-\mathrm{W}$ ones.

In addition from a blind search, at $20-30 \mathrm{GHz}$ it is possible to use lower-frequency catalogs of point sources as tracers for detection [10]. As a result, the source population at such frequencies is much better characterized than the one at higher frequencies. By considering flux number counts at low frequencies it is possible to give an estimate of the flux above which the detected point sources create a complete catalog. This flux threshold is estimated to be abov e1.1 Jy at $23 \mathrm{GHz}$ (K Band) [10,11], while in the W band $(94 \mathrm{GHz})$ the number counts are not sufficiently well determined to allow such an estimate. An alternative blind search technique applied to the WMAP V and W band data increases the number of sources found in the $\mathrm{V}$ band by $50 \%$ compared to the WMAP team results [12]. These results include some sources that were not contained in the WMAP point source mask. The new sources found, however, do not seem to represent a different population than the ones previously detected. As the WMAP five year data is now available, more work on point source characterization at the frequencies where the $\mathrm{CMB}$ science is derived should be possible.

The actual residual contribution of point sources depends upon the mask applied to the observed map. In the case of WMAP, this mask considers the WMAP detected sources (about 300) as well as some bright sources from other low-frequencies catalogs, for a total of approximately 700 sources masked. The actual selection function that this procedure imposes at the $\mathrm{V}$ and $\mathrm{W}$ bands is poorly understood, and indeed some sources detected by [12] were not masked. However, while the detection threshold implied by this whole procedure is poorly defined, there is good agreement in the residual point sources power spectrum contribution as derived by different authors $[8,12,13]$. This can be approximately translated in a flux threshold of $0.6 \mathrm{Jy}$ in the $\mathrm{Q}$ band [13] and $0.75 \mathrm{Jy}$ in the $\mathrm{V}$ band [12]. For illustrative purposes, we will adopt here an approximate estimate for the flux cutoff of $0.7 \mathrm{Jy}$ in all WMAP bands. In Sec. V we discuss the dependence of our results on this choice.

The Planck satellite will have better resolution and sensitivity, resulting in a lower detection threshold for point sources. In the following, we make predictions of the bispectrum expected in the final Planck maps, considering the detection thresholds for a $95 \%$ complete sample derived by [14] using realistic Planck sky simulations. The 
flux cutoffs for both WMAP and Planck, as well as the adopted instrument noise parameters are given in Table I.

Finally, in order to compute the bispectra implied by point sources below a given flux, we adopt the source counts predictions of Toffolatti et al. [15] rescaled by a factor 0.8 , as suggested by the matching of these predictions with the actual number counts at fluxes above 1.1 at $41 \mathrm{GHz}$ obtained by [11]. We will use the radio point source (PS) bias $b^{\mathrm{PS}} \simeq 1.7[16-18]$ as inferred for low frequency radio point sources. This value is uncertain and almost definitely varies for the various population type that constitute the high frequency sample.

It is difficult to constrain the redshift distribution due to the lack of optical studies of $60-96 \mathrm{GHz}$ source population, work at $23 \mathrm{GHz}$ has been conducted by González et al. for fluxes above $1 \mathrm{Jy}$ [11]. At these fluxes and frequencies the number counts are dominated by quasistellar objects for relatively high redshifts. This population is well approximated by the following analytical formula

$$
n^{\mathrm{PS}}(z) \propto 0.75 \times e^{-\left(z-z_{0}\right)^{2} /(2 \sigma)^{2}},
$$

with $z_{0}=0.95$ and $\sigma$ is $0.4(0.9)$ for $z<1(z>1)$. This redshift distribution is in agreement with recent studies of radio sources' populations at $90 \mathrm{GHz}$ with ATCA [19]. In addition, there is a small $(10 \%-15 \%)$ of the total population that consists of radio loud galaxies which peaks at much lower redshifts $(z \leq 0.1)$. Given the small number of sources, it is difficult to derive an appropriate fitting formula for this other population. In the following, we will adopt the following distribution of low-redshift sources

$$
n^{\mathrm{PS}}(z) \propto 0.25 \times 10^{-3 z},
$$

that provides a better fit to counts found by González et al. [11]. We will take the sum of Eqs. (10) and (11) with a common proportionality factor determined by requiring $n^{\mathrm{PS}}(z)$ to integrate to unity over the range $z=0$ to $z=$ 3.1. The relative amplitude of the quasistellar objects and radio galaxy contribution to the sources is derived from the optical identifications of González et al. [11]. This fit will be called model 1. González et al. [11] provide a fit to a theoretical model of the luminosity functions of the various source populations as derived from [20]. To understand how the uncertainty in the source redshift distribution affects our results, we also do calculations with the model of González [11]; this will be called model 2.

WMAP only resolves the high-flux sources which are typically dominated by active galactic nucleus. Most likely lower flux sources consist of a different population (e.g. $[20,21])$ and therefore have a different redshift distribution with possibly more weight either at lower or higher redshifts. An analogous redshift analysis on a higher frequencies catalog is strongly needed, but is not available at this time. Future investigations of radio point sources' catalogs plus WMAP and Planck results are higher frequencies will help clarify this issue. For the aims of this paper we take
Eq. (10) to be the redshift distribution at all frequencies and fluxes and keep in mind the potential uncertainty that this assumption introduces.

\section{B. Thermal Sunyaev-Zeldovich effect}

The hot plasma in the intracluster medium will produce temperature anisotropies via Thomson scattering of the incident CMB photons; this is the well-known thermal SZ effect (see Carlstrom et al. [22] for a review). Following the model of Komatsu and Seljak [23,24], the temperature anisotropies produced by the SZ effect can be expressed as an integral over the cluster mass distribution function

$$
\frac{\Delta T}{T}(\hat{\boldsymbol{n}}, \nu)=g_{\nu} \int d z \frac{d V}{d z} \int_{0}^{\infty} d M y(M, z) \frac{d n}{d M}[M, z ; \hat{\boldsymbol{n}} \hat{\chi}(z)],
$$

here the frequency dependence of the thermal SZ effect is given by

$$
g_{\nu}=x \frac{e^{x}+1}{e^{x}-1}-4
$$

where $x=h \nu / k_{B} T_{\mathrm{CMB}}$. The Compton $y$ parameter is related to the line-of-sight integral of the cluster's thermal pressure, $d n / d M$ is the cluster mass function, and the volume element is

$$
\frac{d V}{d z}=\frac{c}{H(z)} \chi^{2}(z),
$$

where $\chi(z)$ is the comoving distance to redshift $z$. The details of the implementation of this model are extensively discussed in [23]. Ignoring clustering terms in the power spectrum, we find the thermal SZ point source power spectrum

$$
C_{\ell}^{\mathrm{SZ}}(\nu)=g_{\nu}^{2} \int d z \frac{d V}{d z} \int_{0}^{\infty} d M y_{\ell}^{2}(M, z) \frac{d n}{d M}(M, z) .
$$

In a similar fashion to the radio point sources, the number density of massive clusters will be changed by both the biasing effect of the large scale matter overdensity and gravitational lensing magnification. In order to determine how these processes will affect the SZ power spectrum we need to know the redshift distribution of power in the SZ effect. The weighted redshift distribution of power produced by the SZ effect can be expressed as the integral over halo mass of the Sheth-Tormen [25] halo mass function

$$
n^{\mathrm{SZ}}(z) \propto \frac{d V}{d z} \int_{M_{\min }}^{M_{\max }} d M M^{2 \alpha} \frac{d n}{d M}(M, z) .
$$

The cluster $y$ parameter mass scaling relationship slope is taken to be $\alpha=1.6$ [26]. The limits of integration are taken to be $M_{\min }=10^{14} M_{\odot}$ and $M_{\max }=5 \times 10^{15} M_{\odot}$. The redshift distribution is normalized so it will integrate 
to unity. We also need the bias weighted redshift distribution of power produced by the SZ effect which can be expressed as the integral over the Sheth-Tormen halo mass function

$$
(b n)^{\mathrm{SZ}}(z) \propto \frac{d V}{d z} \int_{M_{\min }}^{M_{\max }} d M M^{2 \alpha} \frac{d n}{d M}(M, z) b(M, z),
$$

where $b(M, z)$ is the standard bias of the Sheth-Tormen mass function.

\section{Integrated Sachs-Wolfe effect}

Most of the power in the CMB temperature anisotropies is produced at high redshift during recombination. There will be very little cross correlation between these temperature anisotropies and the low-redshift matter overdensity responsible for altering the small scale point source power spectrum. However additional CMB temperature anisotropies can be generated at low redshift via the ISW effect if the gravitational potential fluctuations are evolving in time. In a fully matter dominated regime the gravitational potential fluctuations are static, however as the Universe becomes dark energy dominated the ISW effect can occur.

The temperature anisotropy produced by the ISW effect can be expressed as

$$
\frac{\Delta T}{T}(\hat{\boldsymbol{n}})=-2 \int d z \frac{\partial \Phi}{\partial z}
$$

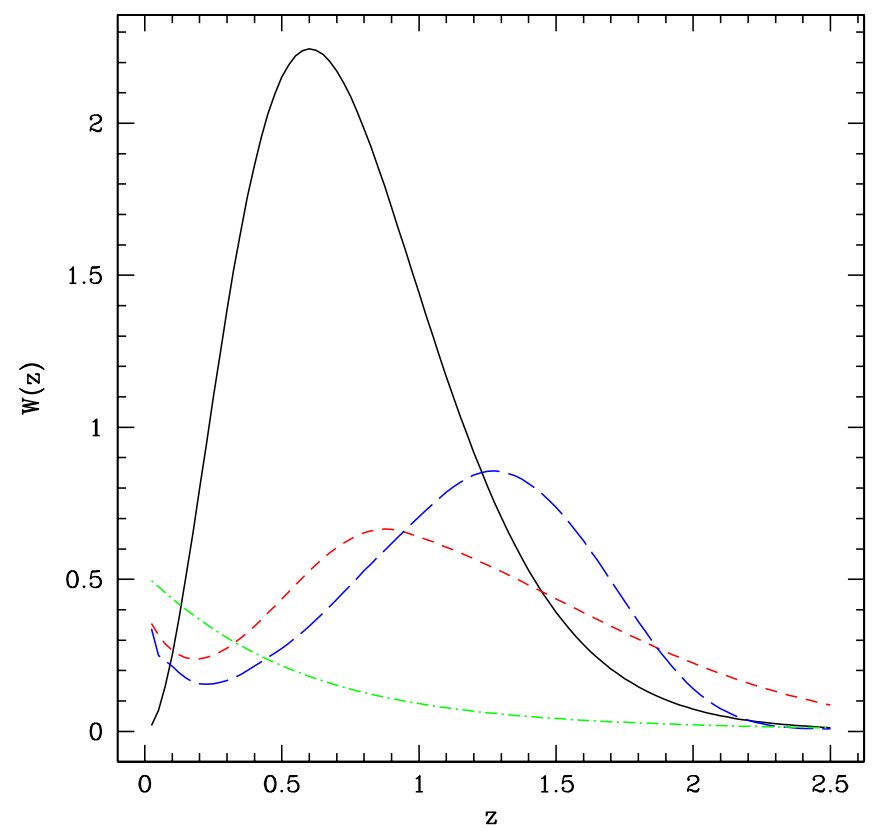

FIG. 1 (color online). Redshift weight functions for the ISW effect (green, dot-dashed line); radio point sources-model 1 (red, dashed line) and model 2 (blue, long-dashed line); and thermal SZ effect (solid, black line).

$$
=3 H_{0}^{2} \Omega_{M} \int d z[(1+z) D(z)]^{\prime} \int \frac{d^{3} \boldsymbol{k}}{(2 \pi)^{3}} e^{i \boldsymbol{k} \cdot \hat{\boldsymbol{n}} \chi} \frac{\delta(\boldsymbol{k})}{k^{2}},
$$

where $D(z)$ is the linear theory growth function and the prime denotes differentiation with respect to $z$.

In Fig. 1 we show the redshift weight functions for the ISW effect $[(1+z) D(z)]^{\prime}$ (green, dot-dashed line); the radio point sources $b^{\mathrm{PS}} n^{\mathrm{PS}}(z) D(z)$-model 1 (red, dashed line) and model 2 (blue, long-dashed line); and the thermal $\mathrm{SZ}$ effect $(b n)^{\mathrm{SZ}}(z) D(z)$ (solid, black line). The overlap of these weight functions will determine the amplitude of the cross correlation spectra as described in Sec. IV B and IV C and shown in Figs. 2 and 5.

\section{Point source bispectrum}

The simplest bispectrum form produced in the CMB is due to radio point source Poisson fluctuations. The reduced bispectrum is independent of scale and can be written as

$$
b_{\ell_{1}, \ell_{2}, \ell_{3}}=c_{\nu}^{-3} \int_{0}^{\bar{S}} d S S^{3} \frac{\overline{d N}}{d S}(S, \nu) .
$$

This bispectrum component has been detected in the WMAP data [27]. Since its functional form is not similar to the local model it will not significantly bias the estimator and we will ignore it as did Yadav and Wandelt. The five year WMAP analysis [3] includes estimates (and corrections) for the estimator bias produced by this bispectrum form.

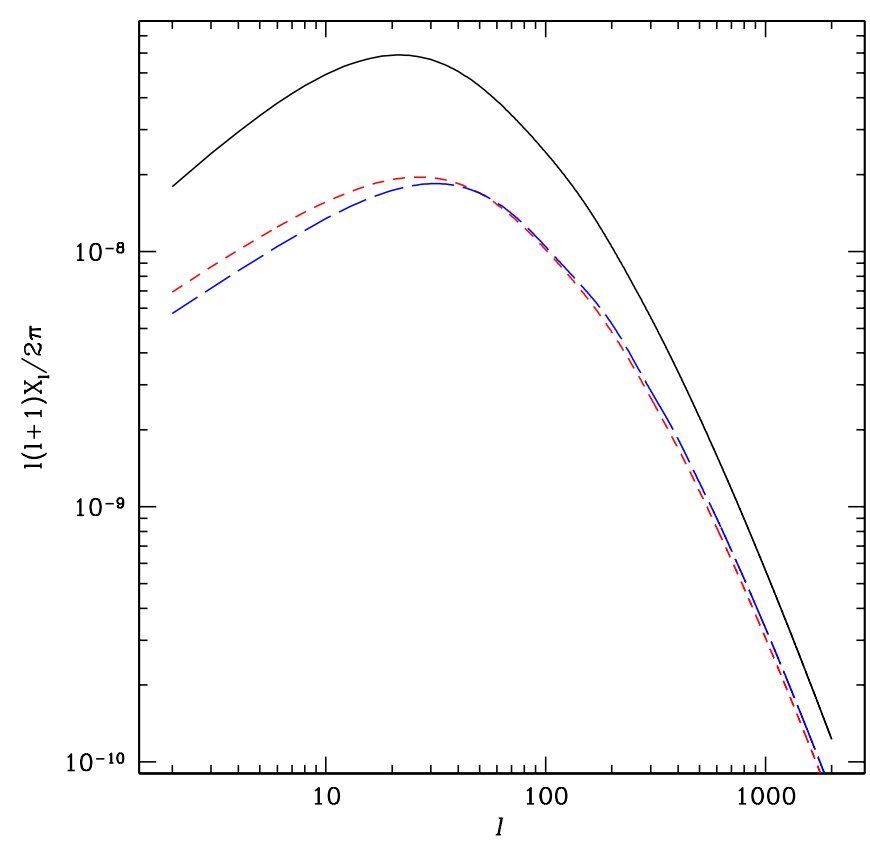

FIG. 2 (color online). Density-ISW cross correlation spectrum for thermal SZ (solid, black line); radio point sources-model 1 (red, dashed line) and model 2 (blue, long-dashed line). 


\section{Number density modulation}

The Poisson fluctuation power spectrum in a certain region of the sky can be written as an integral over the distribution of sources in that region. If the anisotropic component of the power spectrum correlates with any other signal present in the data, non-Gaussian correlation functions will be generated. In this subsection we will focus on correlation of the large scale matter overdensity, which biases the number density of point sources, with the ISW effect.

The power spectrum produced by radio point sources in direction $\hat{\boldsymbol{n}}$ is

$$
C^{\mathrm{PS}}(\hat{\boldsymbol{n}})=c_{\nu}^{-2} \int_{0}^{\bar{S}} d S S^{2} \frac{d N}{d S}(S, \nu ; \hat{\boldsymbol{n}}),
$$

where the anisotropic point source distribution can be expressed in terms of the matter overdensity as

$$
\frac{d N}{d S}(S, \nu ; \hat{\boldsymbol{n}})=\frac{\overline{d N}}{d S}(S, \nu)\left[1+b^{\mathrm{PS}} \int d z n^{\mathrm{PS}}(z) \delta(\hat{\boldsymbol{n}}, z)\right],
$$

the mean point source distribution was described in Sec. III. The large scale matter overdensity, which biases the local number density of point sources as shown in Eq. (22), will be correlated with the large scale CMB temperature anisotropies produced by the ISW effect and the following reduced bispectrum will be induced

$$
b_{\ell_{1}, \ell_{2}, \ell_{3}}=2 \bar{C}^{\mathrm{PS}}\left(X_{\ell_{1}}^{\mathrm{PS}}+\mathrm{cyc}\right) \text {. }
$$

Here the isotropic source distribution $\overline{d N} / d S$ leads to an isotropic power spectrum $\bar{C}^{\mathrm{PS}}$. The matter-ISW cross correlation spectrum can be expressed as

$$
\begin{aligned}
X_{\ell}^{\mathrm{PS}}= & \frac{3 H_{0}^{2} \Omega_{M} b^{\mathrm{PS}}}{\ell^{2}} \int \frac{H(z) d z}{c} P\left[\frac{\ell}{\chi(z)}\right] \\
& \times D(z) n^{\mathrm{PS}}(z)[(1+z) D(z)]^{\prime}
\end{aligned}
$$

where $P(k)$ is the matter power spectrum and $b^{\mathrm{PS}} \simeq 1.7$ is the radio point source bias. The cross correlation spectrum is positive because matter overdensities correspond to potential wells. At low redshift as the amplitude of the potential wells decay the CMB photons experience a net blueshift, thus the cross correlation is positive. In Eq. (24) we have employed Limber's approximation to simplify the cross correlation spectrum (see [28] for an overview of using Limber's approximation to calculate cross correlation spectra). The function $n^{\mathrm{PS}}(z)$ is the redshift probability distribution function of the radio point sources defined in Eq. (10). The large scale matter overdensity affects the thermal SZ power spectrum in an analogous fashion.

In Fig. 2 we show the matter-ISW cross correlation spectrum for the thermal SZ effect (solid, black line) and the radio point sources-model 1 (red, dashed line), model 2 (blue, long-dashed line). The thermal SZ effect has the largest cross correlation because the clusters tend to

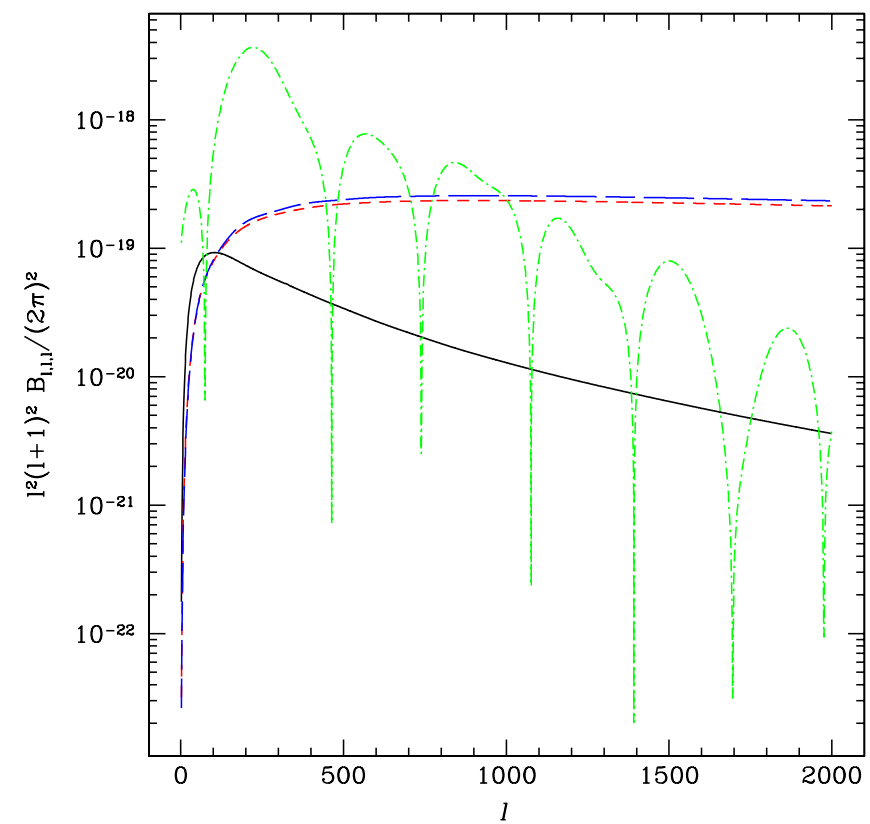

FIG. 3 (color online). Number density modulation equilateral bispectra for thermal SZ (solid, black line); radio point sources-model 1 (red, dashed line) and model 2 (blue, longdashed line). The local model $\left(f_{N L}=1\right)$ (green, dot-dashed line) is also shown for reference. The equilateral shape corresponds to $\left(\ell_{1}=\ell_{2}=\ell_{3}=\ell\right)$.

be located at lower redshift than the radio point sources. The ISW effect primarily occurs at low redshift once the Universe is strongly dark energy dominated, so it most strongly overlaps with the SZ effect.

In Fig. 3 we show the number density modulation equilateral bispectra $\left(\ell_{1}=\ell_{2}=\ell_{3}=\ell\right)$ for the thermal SZ effect (solid, black line) and the radio point sourcesmodel 1 (red, dashed line), model 2 (blue, long-dashed line). Also shown for reference is the primordial local model bispectrum $\left(f_{N L}=1\right)$ (green, dot-dashed line). In Fig. 4 we show a representative collapsed shape $\left(\ell_{1}=5\right.$, $\ell_{2}=\ell_{3}=\ell$ ) for the same bispectra. The equilateral shape of the local model bispectrum changes signs, the zero crossing are obvious from the plot. This is a consequence of the radiative transfer functions producing both hot and cold regions on the sky. The collapsed shape always has the same sign, opposite the sign of $f_{N L}$. For the local model the collapsed bispectra have the highest signal-to-noise.

\section{Magnification modulation}

The distribution of matter along the line-of-sight between the observer and the sources will gravitationally lens these point sources. The principle effect of gravitational lensing will be to change the source density by magnifying and demagnifying certain regions of the sky. The magnification in a given direction can be written, to first order, as 


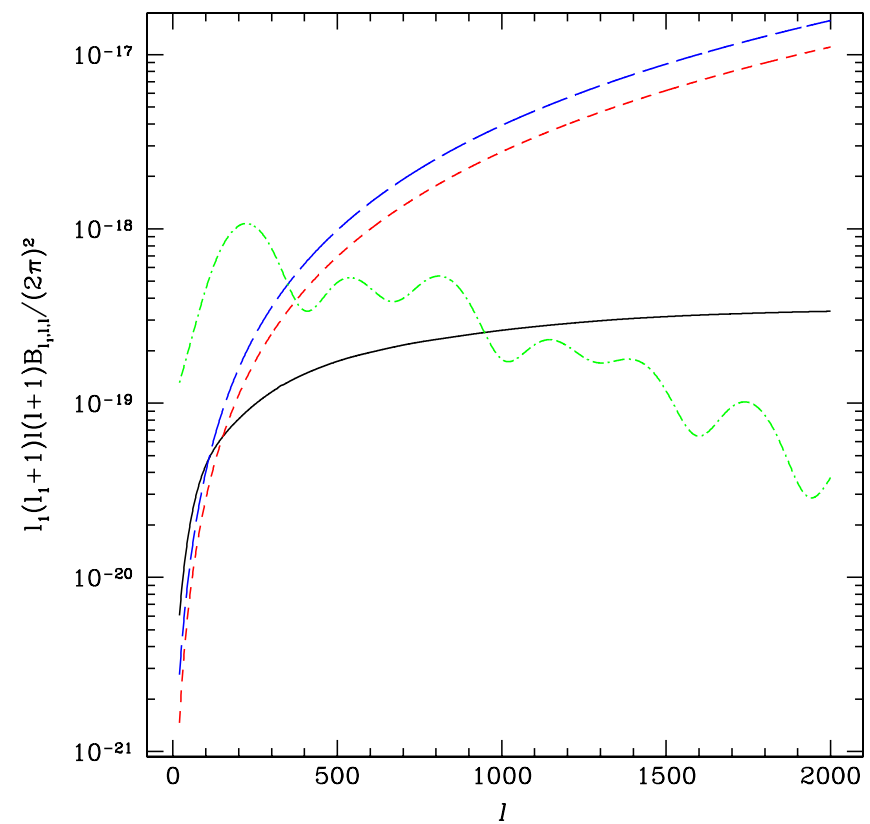

FIG. 4 (color online). Number density modulation collapsed bispectra for thermal SZ (solid, black line); radio point sources-model 1 (red, dashed line) and model 2 (blue, longdashed line). The local model $\left(f_{N L}=1\right)$ (green, dot-dashed line) is also shown for reference. We show representative collapsed bispectra with $\ell_{1}=5, \ell_{2}=\ell_{3}=\ell$.

$$
\mu(\hat{\boldsymbol{n}}) \simeq 1+2 \kappa(\hat{\boldsymbol{n}})
$$

The convergence field distorting a background source at $z$ is related to matter overdensity as

$$
\begin{aligned}
\kappa(\hat{\boldsymbol{n}}, z)= & \frac{3 \Omega_{M} H_{0}^{2}}{2} \int \frac{c d z^{\prime}}{H\left(z^{\prime}\right)}\left(1+z^{\prime}\right) \frac{\chi\left(z^{\prime}\right)}{\chi(z)}\left[\chi(z)-\chi\left(z^{\prime}\right)\right] \\
& \times \delta\left(\hat{\boldsymbol{n}}, z^{\prime}\right)
\end{aligned}
$$

and the average magnification of SZ point sources in a given direction is then given by

$$
\kappa(\hat{\boldsymbol{n}})=\int d z n^{\mathrm{SZ}}(z) \kappa(\hat{\boldsymbol{n}}, z)
$$

The observed SZ cluster number density in a given direction will be related to the intrinsic number density as

$$
\begin{gathered}
\frac{d n^{\mathrm{obs}}}{d M}(M, \hat{\boldsymbol{n}})=\frac{1}{\mu(\hat{\boldsymbol{n}})} \frac{d n}{d M}(M, \hat{\boldsymbol{n}}), \\
\simeq[1-2 \kappa(\hat{\boldsymbol{n}})] \frac{d n}{d M}(M, \hat{\boldsymbol{n}}) .
\end{gathered}
$$

The convergence field is correlated with the CMB temperature anisotropies produced via the ISW effect,

$$
\begin{aligned}
M_{\ell}^{\mathrm{SZ}}= & \frac{9 \Omega_{M}^{2} H_{0}^{4}}{2 \ell^{2}} \int_{0}^{\infty} d z n^{\mathrm{SZ}}(z) \int_{0}^{z} d z_{1} P\left[\frac{\ell}{\chi\left(z_{1}\right)}\right]\left(1+z_{1}\right) \\
& \times D\left(z_{1}\right)\left[\left(1+z_{1}\right) D\left(z_{1}\right)\right]^{\prime} \frac{\chi\left(z_{1}\right)}{\chi(z)}\left[\chi(z)-\chi\left(z_{1}\right)\right] .
\end{aligned}
$$

Again we have evaluated the cross correlation according to Limber's approximation and the convergence-ISW cross correlation spectrum is positive. This magnification effect will result in the following reduced bispectrum

$$
\begin{aligned}
b_{\ell_{1}, \ell_{2}, \ell_{3}}= & -2\left[M_{\ell_{1}}^{\mathrm{SZ}}\left(C_{\ell_{2}}^{\mathrm{SZ}}+C_{\ell_{3}}^{\mathrm{SZ}}\right)+M_{\ell_{2}}^{\mathrm{SZ}}\left(C_{\ell_{1}}^{\mathrm{SZ}}+C_{\ell_{3}}^{\mathrm{SZ}}\right)\right. \\
& \left.\times M_{\ell_{3}}^{\mathrm{SZ}}\left(C_{\ell_{1}}^{\mathrm{SZ}}+C_{\ell_{2}}^{\mathrm{SZ}}\right)\right] .
\end{aligned}
$$

The bispectrum is negative because large scale CMB hot spots (positive ISW effect) correlate with positive magnification which always reduces the amplitude of point source Poisson fluctuations.

The radio point source selection function is expressed in terms of the observed flux which can be affected by gravitational lensing due matter along the line-of-sight. The gravitational lensing magnification will modulate the flux cutoff and be correlated with the ISW temperature anisotropies. The point source dilution effect discussed above will also occur. The radio point source Poisson fluctuation power spectrum can be expressed as

$$
C^{\mathrm{PS}}(\hat{\boldsymbol{n}})=c_{\nu}^{-2} \int_{0}^{\bar{S} / \mu(\hat{\boldsymbol{n}})} d S S^{2} \frac{1}{\mu(\hat{\boldsymbol{n}})} \frac{d N}{d S}(S, \nu) .
$$

Linearizing in the convergence field, we find that the Poisson fluctuation power spectrum becomes anisotropic

$$
C^{\mathrm{PS}}(\hat{\boldsymbol{n}})=2 c_{\nu}^{-2} \kappa(\hat{\boldsymbol{n}})\left[\bar{S}^{3} \frac{d N}{d S}(\bar{S}, \nu)+\bar{C}^{\mathrm{PS}}\right] .
$$

This will result in the following reduced bispectrum

$$
b_{\ell_{1}, \ell_{2}, \ell_{3}}=-4\left(M_{\ell_{1}}^{\mathrm{PS}}+\mathrm{cyc}\right)\left[\bar{S}^{3} \frac{d N}{d S}(\bar{S}, \nu)+\bar{C}^{\mathrm{PS}}\right] \text {, }
$$

where $M_{\ell}^{\mathrm{PS}}$ is the radio point source version of Eq. (30). Note that gravitational lensing affects the radio point source power spectrum by changing both the upper flux cutoff and the source counts, whereas the SZ power spectrum is only altered by changes in the local cluster counts. If the SZ clusters are detected with high signal-to-noise and removed from the CMB maps then flux cutoff modulation effect will also produce an additional bispectrum term.

In Fig. 5 we show the convergence-ISW cross correlation spectrum for the thermal SZ effect (solid, black line) and the radio point sources-mode 1 (red, dashed line), model 2 (blue, long-dashed line). The model 2 of the radio point sources has the largest cross correlation because it predicts that the point sources tend to be located at higher redshift. As opposed to the matter-ISW cross correlation, which requires that the sources lie in the same redshift 
DANIEL BABICH AND ELENA PIERPAOLI

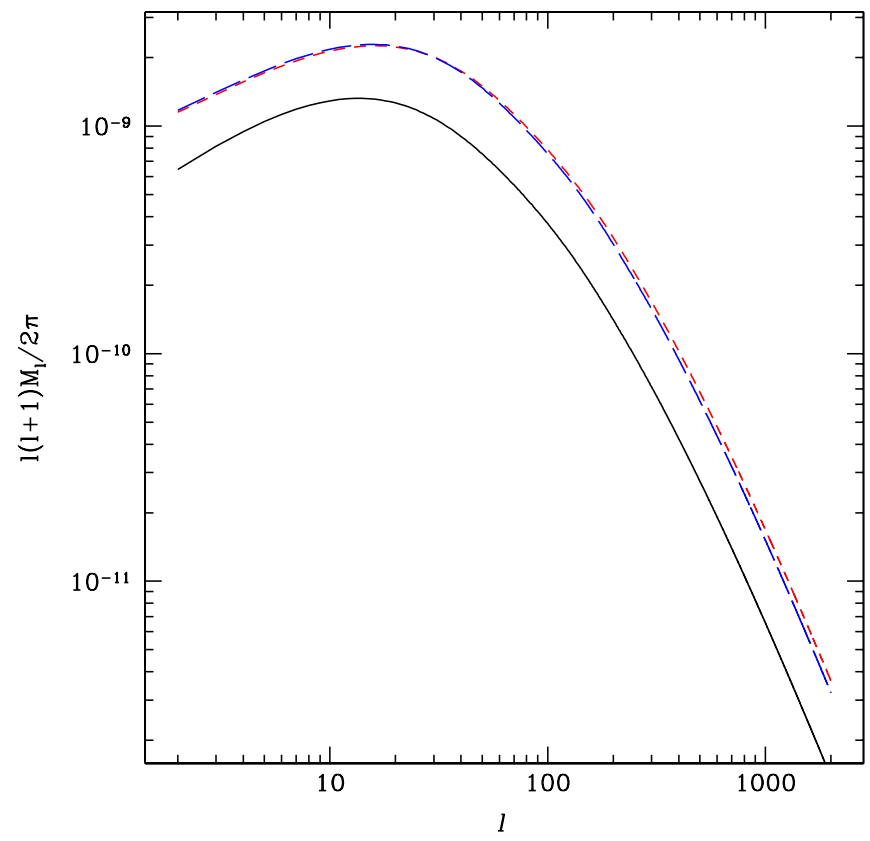

FIG. 5 (color online). Convergence-ISW cross correlation spectrum for thermal SZ (solid, black line); radio point sources-model 1 (red, dashed line) and model 2 (blue, longdashed line).

range over which the ISW effect occurs, in this case the point source do not have to be at the same redshift at which the ISW occurs in order for the contribution to be relevant.

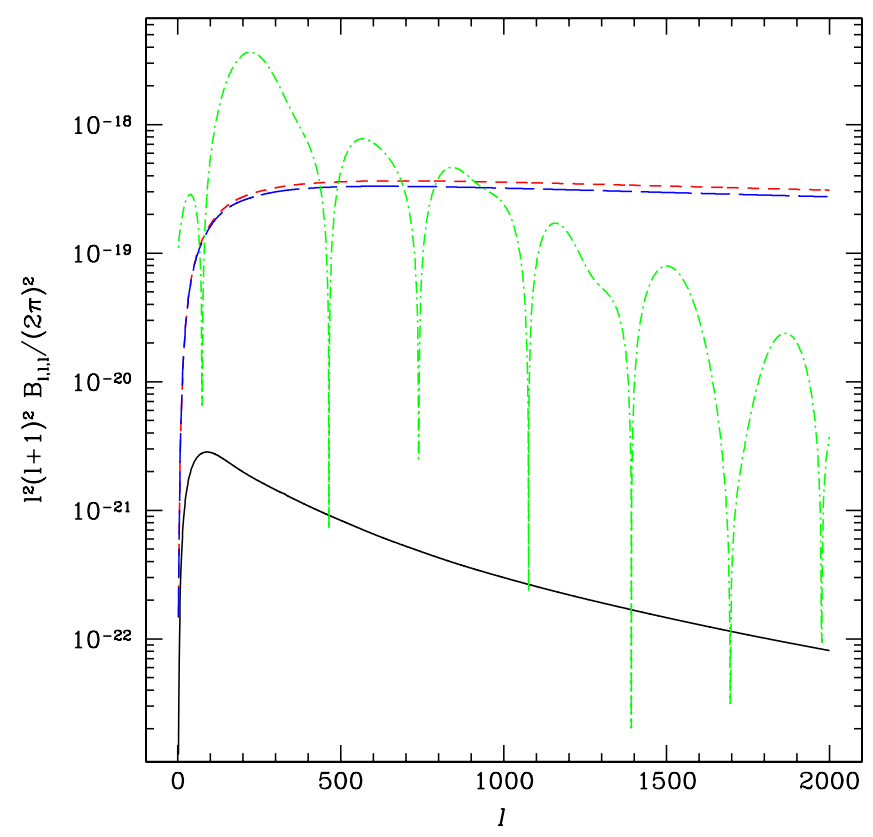

FIG. 6 (color online). Magnification modulation equilateral bispectra for thermal SZ effect (solid, black line); radio point sources-model 1 (red, dashed line) and model 2 (blue, longdashed line). The local model bispectrum $\left(f_{N L}=1\right)$ (green, dotdashed line) is also shown for reference.
PHYSICAL REVIEW D 77, 123011 (2008)

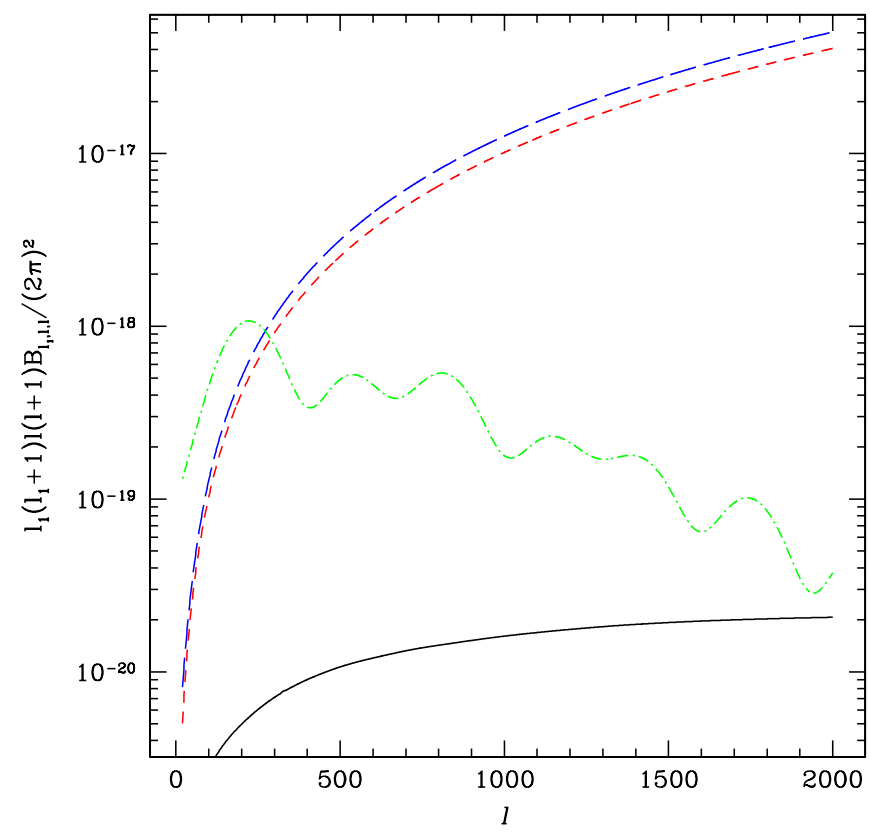

FIG. 7 (color online). Magnification modulation collapsed bispectra for thermal SZ (solid, black line); radio point sourcesmodel 1 (red, dashed line) and model 2 (blue, long-dashed line). The local model $\left(f_{N L}=1\right)$ (green, dot-dashed line) is also shown for reference. We show representative collapsed bispectra with $\ell_{1}=5, \ell_{2}=\ell_{3}=\ell$.

In fact the point sources experience greater magnification if they are at substantially higher redshift than the matter distribution that is simultaneously magnifying them and is correlated with the ISW effect.

In Fig. 6 we show the magnification modulation equilateral bispectra for the thermal SZ effect (solid, black line); radio point sources-model 1 (red, dashed line) and model 2 (blue, long-dashed line). The local model bispectrum $\left(f_{N L}=1\right)$ (green, dot-dashed line) is also shown for reference. In Fig. 7 we show a representative collapsed shape $\left(\ell_{1}=5, \ell_{2}=\ell_{3}=\ell\right)$ for the same bispectra.

\section{Selection modulation}

The amplitude of the Poisson fluctuation power spectrum depends on the number density of radio point sources below some flux limit determined by the radio point source removal technique. If the selection criterion used produces an anisotropic flux limit that is correlated with either the large scale temperature anisotropy or the instrument noise, then a bispectrum will be produced. If the radio point sources are identified via external catalogs, such as NVSS or PMN, then there will be no cross correlation and therefore no bispectrum. And if multiwavelength data is differenced to isolate the power-law frequency dependence of the radio point source, then the modulated selection function will produce correlations between the data in different frequency bands. 
The simplest method is to removal pixels above some multiple $(\gamma)$ of the pixel variance

$$
\bar{S}(\hat{\boldsymbol{n}})=c_{\nu}\left[\gamma \sigma_{p}-\Delta \Omega\left(\frac{\Delta T}{T}(\hat{\boldsymbol{n}})+N(\hat{\boldsymbol{n}})\right)\right] .
$$

The temperature anisotropy and instrument noise have vanishing expectation values, so the mean flux cutoff is

$$
\bar{S}_{0}=c_{\nu} \gamma \sigma_{p},
$$

where the pixel variance is defined as

$$
\sigma_{p}^{2}=(\Delta \Omega)^{2} \sum_{\ell} \frac{(2 \ell+1)}{4 \pi} C_{\ell}^{T},
$$

here $C_{\ell}^{T}=C_{\ell}+C_{\ell}^{N}$ is the sum of the CMB signal and noise and $\Delta \Omega$ is the pixel size. There will be fluctuations about the expected flux cutoff that are correlated with either the temperature anisotropy or the instrument noise. Linearizing in these fluctuations, we find the following reduced bispectrum

$$
b_{\ell_{1}, \ell_{2}, \ell_{3}}=-\frac{\Delta \Omega}{c_{\nu}}\left(C_{\ell_{1}}^{T}+\text { cyc }\right) \bar{S}_{0}^{2} \frac{d N}{d S}(\bar{S}, \nu) .
$$

The selection criterion used by WMAP is based on an algorithm developed by Tegmark and de Oliveira-Costa [29]. The map is filtered in order to reduce the importance of the long-wavelength $\mathrm{CMB}$ modes. The filtered total temperature fluctuation in some pixel is

$y(\hat{\boldsymbol{n}})=\sum_{\ell m} \frac{1}{C_{\ell}^{T}} Y_{\ell m}(\hat{\boldsymbol{n}})\left(a_{\ell m}+n_{\ell m}\right) \Delta \Omega+\sum_{\ell} \frac{(2 \ell+1)}{4 \pi} \frac{S(\hat{\boldsymbol{n}})}{c_{\nu} C_{\ell}^{T}}$.

The algorithm removes any pixel that has a value greater than some multiple $(\gamma)$ of the filtered map pixel variance

$$
\tilde{\sigma}_{p}^{-2}=(\Delta \Omega)^{-2} \sum_{\ell} \frac{(2 \ell+1)}{4 \pi} \frac{1}{C_{\ell}^{T}} .
$$

The threshold $\gamma$ is chosen according to some compromise between false positive and negatives. Since the thresholding is applied to the total signal in a pixel, the corresponding radio point source flux cutoff in a pixel is

$$
\bar{S}(\hat{\boldsymbol{n}})=\frac{c_{\nu}}{F}\left[\gamma \tilde{\sigma}_{p}-\Delta \Omega \sum_{\ell m} \frac{1}{C_{\ell}^{T}} Y_{\ell m}(\hat{\boldsymbol{n}})\left(a_{\ell m}+n_{\ell m}\right)\right],
$$

where the normalization is

$$
F=\sum_{\ell} \frac{(2 \ell+1)}{4 \pi} \frac{1}{C_{\ell}^{T}} .
$$

The temperature anisotropy and instrument noise have vanishing expectation values, so the mean flux cutoff is

$$
\bar{S}_{0}=\frac{c_{\nu}}{F} \gamma \tilde{\sigma}_{p} .
$$

There will be fluctuations about this expected value and these fluctuations will be correlated with either the temperature anisotropy or the instrument noise. Linearizing in these fluctuations, we find the following reduced bispectrum

$$
b_{\ell_{1}, \ell_{2}, \ell_{3}}=-\frac{3 \Delta \Omega}{c_{\nu} F} \bar{S}_{0}^{2} \frac{d N}{d S}\left(\nu, \bar{S}_{0}\right) .
$$

In regions with large instrument noise or CMB temperature anisotropy, the radio point source flux cutoff will be lowered. This reduces the total number density of radio point sources in that region and therefore the Poisson fluctuation power spectrum. This effect explains the negative sign in the reduced bispectrum, Eq. (44).

Since the filtering applied to the maps produces a bispectrum independent of scale, similar to the point source bispectrum described in Sec. IVA, we will ignore it. In reality the actual selection function is more complicate than this simple filter technique predicts and it might produce a bispectrum of a much different form. Numerical simulations incorporating the exact selection procedure will need to be done in order to fully determine its effect on the estimator.

\section{NUMERICAL RESULTS}

In this section we will present numerical results for the contamination of the standard non-Gaussianity estimator by the various bispectra discussed in this paper. This is done for both the WMAP and Planck instrument noise levels, frequency bands, and flux cutoffs given in Table I.

In Fig. 8 the $f_{N L}$ estimator bias as a function of $\ell_{\max }$ is shown for the different bispectra-radio point source number density modulation (solid, black line); SZ number density modulation (dotted, red line); radio point source gravitational lensing magnification modulation (dashed, blue line); and SZ gravitational lensing magnification modulation (long-dashed, green line) with WMAP instrument noise. The bias plots are shown for the four relevant WMAP frequency bands - upper left $\mathrm{Ka}-33 \mathrm{GHz}$; upper right $\mathrm{Q}-40 \mathrm{GHz}$; lower left $\mathrm{V}-61 \mathrm{GHz}$; lower right $\mathrm{W}-94 \mathrm{GHz}$. The magnification modulation effect produces a positive bias since its bispectrum is negative, while the density modulation effect produces a negative bias.

Yadav and Wandelt claim a central value of $f_{N L}=86.8$ with a standard deviation of $\sigma=30.0$ for a $2.9 \sigma$ detection of non-Gaussianity [1]. In the five year WMAP data the central value is found to be $f_{N L}=67$ with a standard deviation of $\sigma=31$ [3]. At $\ell_{\max }=750$ the total estimator bias $\Delta f_{N L}=0.35$ in the Ka band, $\Delta f_{N L}=0.24$ in the Q band, $\Delta f_{N L}=-0.097$ in the $\mathrm{V}$ band, and $\Delta f_{N L}=-0.13$ in the $\mathrm{W}$ band. Since the density modulation and the magnification modulation bispectra have different signs they partially cancel and reduce the overall effect. At low frequency the radio point source magnification modulation bispectrum is the most important so the bias is positive. At 

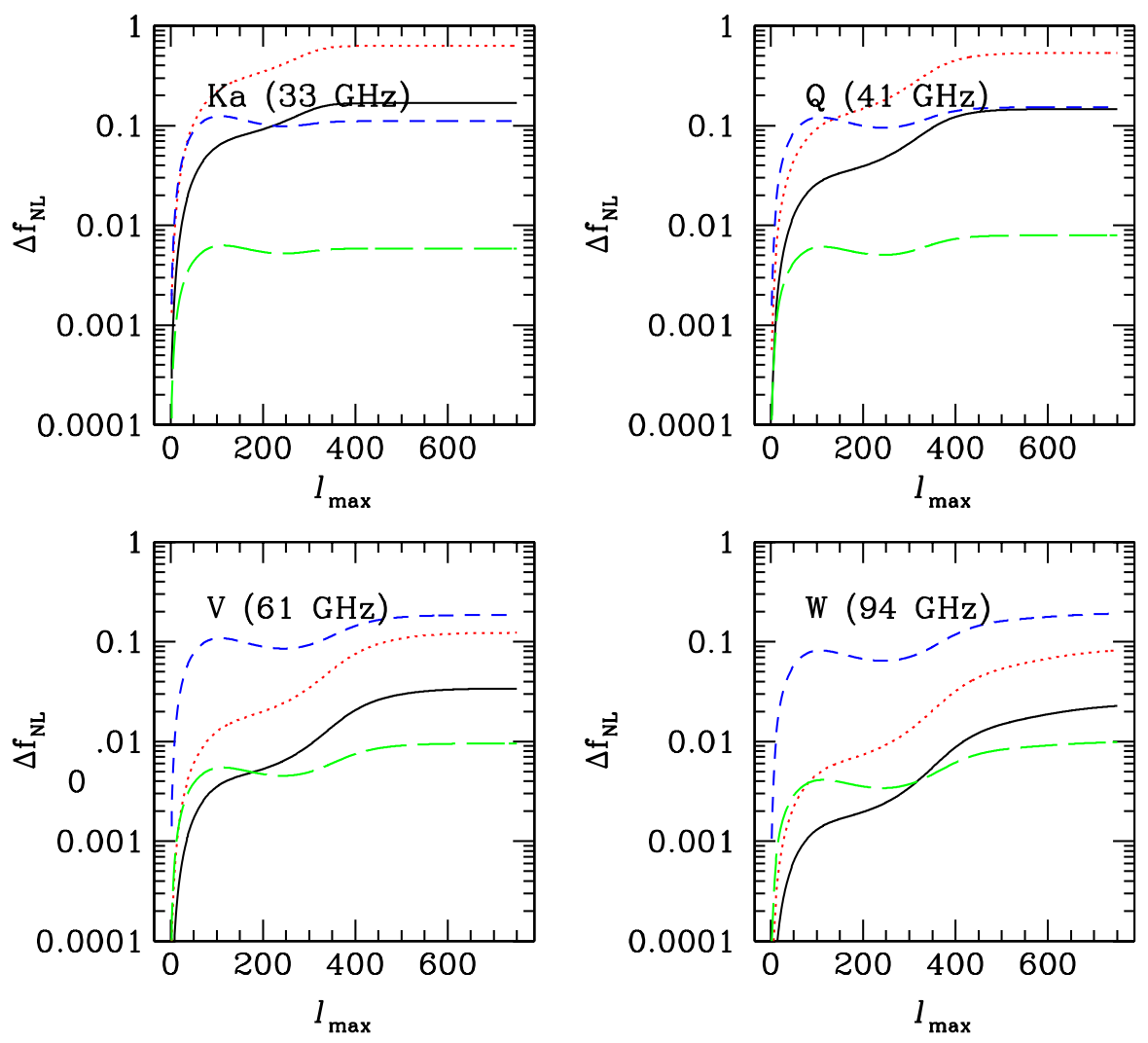

FIG. 8 (color online). The WMAP estimator bias terms $\Delta f_{N L}^{\alpha}$ as a function of $\ell_{\max }$ for radio point source density modulation (solid, black line); SZ number density modulation (dotted, red line); radio point source gravitational lensing magnification modulation (dashed, blue line); and SZ gravitational lensing magnification modulation (long-dashed, green line) in $\mathrm{Ka}-33 \mathrm{GHz}$ (upper left); $\mathrm{Q}-40 \mathrm{GHz}$ (upper right); $\mathrm{V}-61 \mathrm{GHz}$; and $\mathrm{W}-94 \mathrm{GHz}$. The density modulation terms produce a negative bias, while the magnification bias produce a positive bias.

higher frequencies the SZ density modulation bispectrum dominates which makes the bias negative. These numbers should be compared to the estimator bias produced by the radio point source Poisson fluctuation bispectrum. Komatsu et al. [3] have estimated this bias to be $\Delta f_{N L} \simeq$ $-(3-5)$ at $\ell_{\max }=700$.

In Fig. 9 the $f_{N L}$ estimator bias as a function of $\ell_{\max }$ is shown for the different bispectra-radio point source number density modulation (solid, black line); SZ number density modulation (dotted, red line); radio point source gravitational lensing magnification modulation (dashed, blue line); and SZ gravitational lensing magnification modulation (long-dashed, green line) for Planck at $30 \mathrm{GHz}$ (upper left); $44 \mathrm{GHz}$ (upper left); $70 \mathrm{GHz}$ (lower left), and $100 \mathrm{GHz}$ (lower right).

Estimates of the sensitivity on $f_{N L}$ achievable with Planck suggest that $\Delta f_{N L} \simeq 10$ at $95 \%$ C.L. using just temperature information and $\Delta f_{N L} \simeq 5$ at $95 \%$ C.L also including polarization. Summing the various biases we find $\Delta f_{N L}=1.3$ at $\nu=30 \mathrm{GHz}, \quad \Delta f_{N L}=0.34$ at $\nu=$ $44 \mathrm{GHz}, \Delta f_{N L}=-0.25$ at $\nu=70 \mathrm{GHz}$, and $\Delta f_{N L}=$ -0.48 at $\nu=100 \mathrm{GHz}$. These results imply that a good knowledge of point source properties is important if Planck will be able to achieve its full potential in constraining primordial non-Gaussianity.

There are uncertainties in the models we have used to describe the radio point sources. These model uncertainties will directly lead to uncertainties in the above predictions. As can be directly seen in Figs. 2-6, that the differences between the radio point source redshift distributions, model 1 and model 2, are not significant. This is not surprising as the ISW kernel, with which the radio point source redshift distributions are being cross correlated, is quite broad. The redshift distributions are supposed to trace both the high-flux source populations at low frequencies. However, they may not be representative of the source populations at all frequencies considered here for lower flux cut thresholds. If a lower flux cut implies a higher population of low-redshift objects, the amplitude of the ISW-density cross correlation spectrum would be increased. The decrease in the flux cutoff will decrease the Poisson fluctuation power spectrum, so the change in the bias of the $f_{N L}$ estimator is not clear. We also note that reducing the flux cutoff from the WMAP to the Planck level does not reduce the bias implied on $f_{N L}$ as the Planck noise levels and beam sizes are also smaller. 

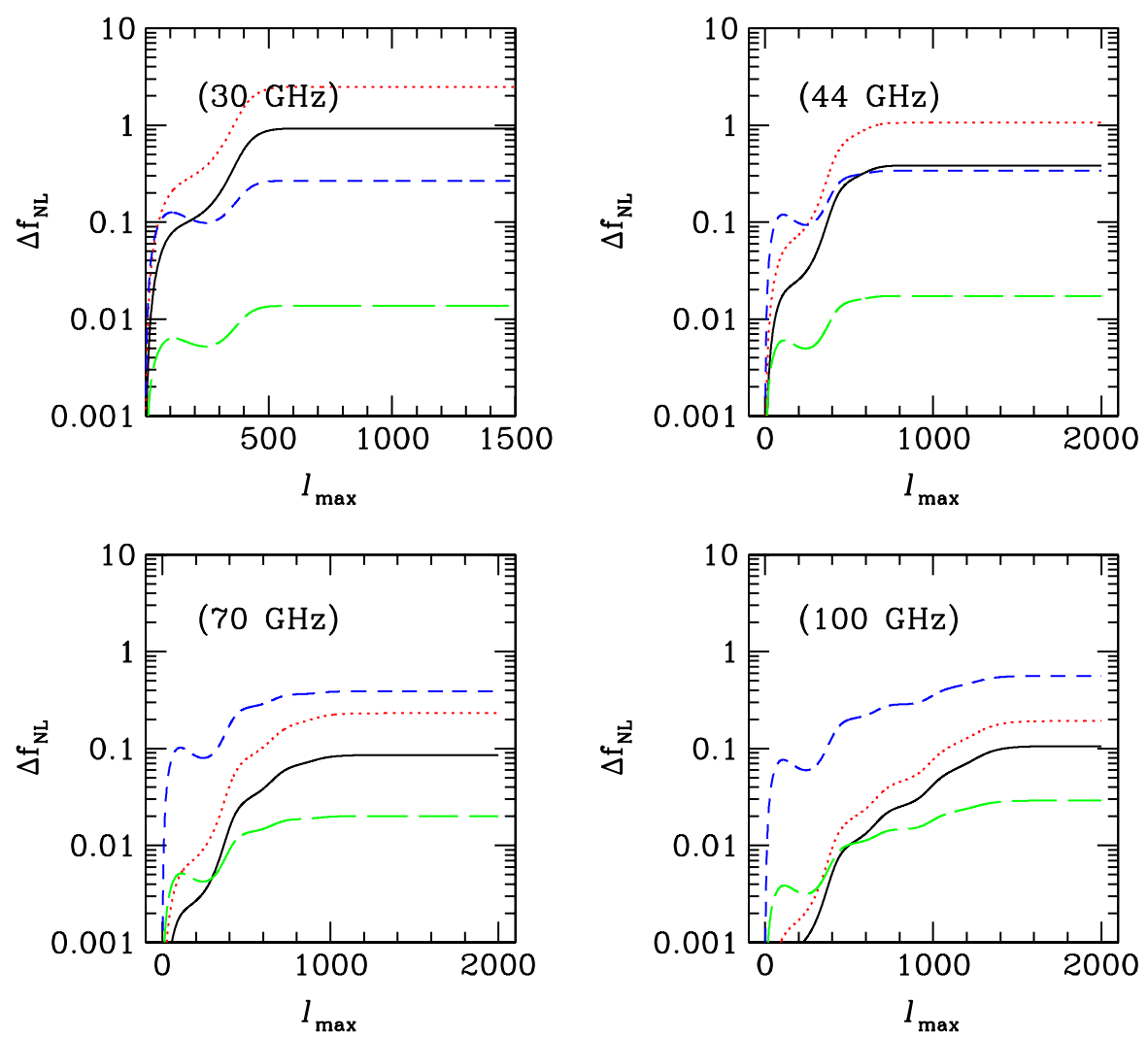

FIG. 9 (color online). The Planck estimator bias terms $\Delta f_{N L}^{\alpha}$ for $30 \mathrm{GHz}$ (upper left); $44 \mathrm{GHz}$ (upper right); $70 \mathrm{GHz}$ (lower left), and $100 \mathrm{GHz}$ (lower right). The curves are the same as Fig. 8. The density modulation terms produce a negative bias, while the magnification bias produce a positive bias.

\section{CONCLUSION}

In this paper we analyzed the effect of point sources, both due to radio emission and the cluster SZ effect, on the estimation of primordial non-Gaussianity in the cosmic microwave background. The standard non-Gaussianity estimator is sensitive to any bispectrum present in the data. In addition to the standard Poisson fluctuation bispectrum, we found that cross correlations between the radio point source and SZ power spectra and either the CMB temperature anisotropies or instrument noise can produce bispectra. These bispectra have forms somewhat similar to the local model, which is the standard bispectrum form used to search for primordial non-Gaussianity in CMB data. These similarities are not accidental, but occur because the same basic principle generates the non-Gaussianity in these cases. Because of this similarity it will be much more difficult to distinguish this non-Gaussianity from the primordial signal than other secondary bispectra with shapes which can be quite different.

A related paper by Serra and Cooray [30] has examined different secondary bispectra, but has reached similar conclusions. They examined the bispectra produced by the cross correlation of the thermal SZ effect and the gravitational lensing of the primary $\mathrm{CMB}$ anisotropies. They concluded that the effects are too small to account for the inferred values of $f_{N L}$ from the WMAP data and will start to become important for the Planck data set. The estimator biases calculated in this paper are generally smaller than the bias produced by the radio point source Poisson fluctuation bispectrum despite the fact that Poisson fluctuations produce a bispectrum with a dissimilar shape when compared to the local model. The overall amplitude of the ISW effect is quite small which means that the amplitudes of both the cross correlation spectra and the bispectra calculated in this paper are also very small. The amplitude of the radio point source Poisson fluctuation bispectrum is much larger than any of the bispectra calculated in this paper and still dominates the estimator bias despite the difference in bispectrum shape.

The estimator bias that we have calculated is small and is not able to explain the results found by Yadav and Wandelt, although the bispectra considered in our paper will start to become important for the Planck nonGaussianity analysis. We should emphasize that if Planck detects a large value of $f_{N L}$ the various estimator biases we discuss in this paper, and others that are discussed elsewhere, will not change the qualitative interpretation of that result. However a correction to the estimator's central value due to the estimator's bias will be required. This 
correction will be comparable to the estimator's statistical uncertainty which will be much smaller than the value claimed by Yadav and Wandelt for Planck data.

\section{ACKNOWLEDGMENTS}

D. B. acknowledges financial support from the Betty and Gordon Moore Foundation and would like to thank Sterl Phinney, Daisuke Nagai, and Eiichiro Komatsu for helpful conversations. E. P. is supported by NSF Grant No. AST0649899,NASA Grant No. NNX07AH59G, Planck Subcontract No. 1290790, and JPL SURP No. 1314616. She would also like to thank Kevin Huffenberger and Joachin Gonzales-Nuevo for useful conversations. We also thank Kendrick Smith for helpful comments on a draft of the paper.
[1] A. P. S. Yadav and B. D. Wandelt, arXiv:0712.1148.

[2] D. N. Spergel, R. Bean, O. Doré, M. R. Nolta, C. L. Bennett, J. Dunkley, G. Hinshaw, N. Jarosik, E. Komatsu, L. Page et al., Astrophys. J. Suppl. Ser. 170, 377 (2007).

[3] E. Komatsu, J. Dunkely, M. R. Nolta, C. L. Bennett, M. Gold, G. Hinshaw, N. Jarosik, M. Limon, D. Larson et al., arXiv:astro-ph/0803.0547 [Astrophys. J. Suppl. Ser. (to be published)].

[4] J. Maldacena, J. High Energy Phys. 05 (2003) 13.

[5] E. Komatsu, arXiv:astro-ph/0206039 [Astrophysics (Engl. Transl.) (to be published)].

[6] D. Babich, Phys. Rev. D 72, 043003 (2005).

[7] P. Creminelli, A. Nicolis, L. Senatore, M. Tegmark, and M. Zaldarriaga, J. Cosmol. Astropart. Phys. 05 (2006) 4.

[8] G. Hinshaw, M. R. Nolta, C. L. Bennett, R. Bean, O. Doré, M. R. Greason, M. Halpern, R. S. Hill, N. Jarosik, A. Kogut et al., Astrophys. J. Suppl. Ser. 170, 288 (2007).

[9] E. Pierpaoli, Astrophys. J. 589, 58 (2003).

[10] M. López-Caniego, J. González-Nuevo, D. Herranz, M. Massardi, J.L. Sanz, G. De Zotti, L. Toffolatti, and F. Argüeso, Astrophys. J. Suppl. Ser. 170, 108 (2007).

[11] J. González-Nuevo, M. Massardi, F. Argüeso, D. Herranz, L. Toffolatti, J.L. Sanz, M. López-Caniego, and G. de Zotti, Mon. Not. R. Astron. Soc. 384, 711 (2008).

[12] X. Chen and E. L. Wright, arXiv:0712.3594.

[13] K. M. Huffenberger, H. K. Eriksen, and F. K. Hansen, Astrophys. J. Lett. 651, L81 (2006).

[14] M. López-Caniego, D. Herranz, J. González-Nuevo, J. L. Sanz, R. B. Barreiro, P. Vielva, F. Argüeso, and L. Toffolatti, Mon. Not. R. Astron. Soc. 370, 2047 (2006).
[15] L. Toffolatti, F. Argueso Gomez, G. de Zotti, P. Mazzei, A. Franceschini, L. Danese, and C. Burigana, Mon. Not. R. Astron. Soc. 297, 117 (1998).

[16] K. M. Smith, O. Zahn, and O. Doré, Phys. Rev. D 76, 043510 (2007).

[17] C. Blake, P. G. Ferreira, and J. Borrill, Mon. Not. R. Astron. Soc. 351, 923 (2004).

[18] S. P. Boughn and R. G. Crittenden, Phys. Rev. Lett. 88, 021302 (2002).

[19] E. M. Sadler, R. Ricci, R. D. Ekers, R. J. Sault, C. A. Jackson, and G. De Zotti, arXiv:0709.3563.

[20] G. de Zotti, R. Ricci, D. Mesa, L. Silva, P. Mazzotta, L. Toffolatti, and J. González-Nuevo, AAS Sci. Technol. Ser. 431, 893 (2005).

[21] E. Pierpaoli and R. Perna, Mon. Not. R. Astron. Soc. 354, 1005 (2004).

[22] J. E. Carlstrom, G. P. Holder, and E. D. Reese, Annu. Rev. Astron. Astrophys. 40, 643 (2002).

[23] E. Komatsu and U. Seljak, Mon. Not. R. Astron. Soc. 336, 1256 (2002).

[24] E. Komatsu and U. Seljak, Mon. Not. R. Astron. Soc. 327, 1353 (2001).

[25] R. K. Sheth and G. Tormen, Mon. Not. R. Astron. Soc. 308, 119 (1999).

[26] D. Nagai, Astrophys. J. 650, 538 (2006).

[27] E. Komatsu, A. Kogut, M. R. Nolta, C. L. Bennett, M. Halpern, G. Hinshaw, N. Jarosik, M. Limon, S. S. Meyer, L. Page et al., Astrophys. J. Suppl. Ser. 148, 119 (2003).

[28] A. Cooray, Phys. Rev. D 65, 103510 (2002).

[29] M. Tegmark and A. de Oliveira-Costa, Astrophys. J. Lett. 500, L83 (1998).

[30] P. Serra and A. Cooray, Phys. Rev. D 77, 107305 (2008). 